\title{
NEW PERSPECTIVE IN STUDYING PROPOFOL AND SLEEP INTERACTION: A PILOT STUDY
}

\author{
By
}

MOHAMED A. MAHER ${ }^{1 *}$, EMAN A. MAHER ${ }^{2}$, AHMED I. REFAAT ${ }^{1}$, MOHAMED M. HUSSEIN ${ }^{1}$, REEHAM S. EBIED ${ }^{1}$, AND AHMED HAZEM HELMY ${ }^{3}$

Department of Anesthesiology ${ }^{1}$, Theodor Bilharz Research Institute, Cairo, Clinical Neurophysiology Unit ${ }^{2}$, Department of Neurology, Faculty of Medicine, Cairo University, Giza, and Department of Surgery ${ }^{3}$, Theodor Bilharz Research Institute, Cairo, Egypt ('Correspondence: d.mmaher@yahoo.com, Fax: +20235408125)

\begin{abstract}
Several studies suggested that propofol satisfied the need for both rapid eye movement (REM) and slow wave sleep (SWS). Most of these studies were based either on animal models or on induced sleep deprivation without further clinical extrapolation. The this preliminary study investigated the relation between natural preoperative sleep (REM and SWS) and operative propofol induction dose, whether or not clinical impact can be extracted from this relation, and finally to assess the feasibility of conducting a similar full-scale trial.

This study was a prospective observational study. 27 ASA I adult male patients scheduled for elective surgery were subjected to preoperatively sleep EEG. Propofol induction dose achieving Bispectral Index (BIS) value between 40 and 60 was recorded. Correlation between propofol dose and both SWS \% and REM \% was done. Patients were classified according to sleep EEG into good sleepers and bad sleepers. Propofol dose in good sleepers and bad sleepers was compared.

The results showed a positive correlation between propofol induction dose and both SWS (\%) $(r=0.532 ; p=0.003)$ and REM $(\%)(r=0.518 ; p=0.005)$. The median value of propofol induction dose in good sleepers was $1.70(1.00-2.00)$, while in bad sleepers it was significantly lower 1.10 $(0.80-1.70)(p$ value $=0.01)$.
\end{abstract}

Keywords: pre-operative sleep, propofol, rapid eye movement, slow wave sleep

\section{Introduction}

General anesthesia produces a state often described by anesthetists and patient as sleep or coma (Brown et al, 2010). Although little is known about how exactly either state is generated and maintained, yet they share a lot and also differ a lot. Unlike sleep general anesthesia is a drug induced dose dependant state of reduced responsiveness, which is minimally altered by environmental factors (Tung and Mendelson, 2004). Also Electroencephalographic (EEG) findings in both states varied widely (Murphy et al, 2011). Besides, recovery of consciousness and cognitive function after sleep and anesthesia show some discrepancies (Kaitlyn et al, 2017).

Sleep and anesthesia share many components. Genetically some hypothesized that a single gene can control both sleep behavior and sensitivity to anesthetic drugs (Weber et $a l, 2009)$. Neurochemically, various anesthetic drugs perform their action at least partly by duplicating activities on neural systems involved in sleep wake regulation (Lydic and Baghdoyan, 2005) (Franks, 2008). PET scanning (Alkire et al, 1999) and microelectrode recordings (Vahle-Hinz et al, 2001) of thalamic relay neuronal activity during anesthesia demonstrate activities similar to those occurring during sleep.

Regarding propofol, several studies investigated the relation between it and sleep. Micro administration of the intravenous anesthetic propofol into the medial preoptic area in rats increases sleep in a dose-dependent fashion (Tung et al, 2001). Another study also on rats proved that sleep deprivation potentiated propofol ability to induce loss of righting reflex (Tung et al, 2002).

In rats too, pre-existing sleep debts accumulated over the previous 24 hours of sleep deprivation dissipate identically under propofol-anesthesia as they would have during recovery sleep (Tung et al, 2004). Moreover, when propofol anesthesia is induced at 
the onset of the rest cycle in rodents and maintained for 12 hours neither REM nor SWS sleep debts occurred (Tung et al 2001). Thus, propofol satisfied the need for both REM and SWS (Chidambaran et al, 2016).

This study aimed to investigate: 1 - the mentioned sleep and propofol relation hypothesis in humans unlike all other studies carried out on rats, 2- propofol sleep relation in its clinical daily use by analyzing natural preoperative sleep (without induced maneuvers) and correlating it to propofol induction dose, 3- to extract a clinical impact from this relation and whether preoperative sleep quality can affect propofol induction dose and 4- to assess the feasibility of conducting a similar full-scale trial.

\section{Subjects and methods}

Design: This study is a prospective observational study.

After having approval of the Ethical Committee of Theodor Bilharz Research Institute and written informed consent; studied 27 ASA I adult male patients scheduled for elective cholecystectomy.

Inclusion criteria: Age range (20-40) years, Body mass index (BMI) 25-30, Regular sleep wake pattern, and Patient with Epworth sleepiness scale scores less than 5 .

Exclusion criteria: History suggestive sleep disorders such as insomnia and excessive daytime sleepiness, Intake of any drugs that could affect sleep or cognition such as stimulants, hypnotics, benzodiazepines and antidepressants, and History or evidence at clinical examination of any chronic systemic disease.

Preoperatively, all patients were subjected to 1-Clinical assessment: history taking, general and neurological examination and laboratory work up to exclude any medical condition.2- Sleep diary: for one week prior to the operation to monitor subjects' sleepwake schedules. 3- Epworth sleepiness scale: which evaluates subjective sleepiness and any patient with score more than 5 were excluded to ensure that all of the patients have nearly the same levels of subjective sleepiness. 4- Sleep Electroencephalography (EEG): for 8 hours the night just before the operation. Software used was Nicolet V.5.3. 21 electrodes were applied to the scalp according to the international 10-20 system.

For Eye movements recording additional 2 electrodes were applied one $\mathrm{cm}$ below the left outer canthus and one cm above the outer canthus respectively. Finally, for muscle tone assessment 2 electrodes were placed over the mandible. The record started as a trial for few minutes to ensure proper calibration. Gain was set as $30 \mu \mathrm{v} / \mathrm{cm}$, paper speed at $30 \mathrm{~mm} / \mathrm{sec}$ and the filter bandwidth was adjusted to $35 \mathrm{~Hz}, 1,600 \mathrm{~Hz}, 50 \mathrm{~Hz}$. EEG was manually scored for the following: 1- Total sleep time (TST), 2- Slow wave sleep\% (SWS), and 3- Rapid eye movement sleep\% (REM).

According to EEG, patients were classified into three groups: G1: Good sleepers were those who have normal sleep EEG values (Ohayon et al, 2004), i.e. TST equal to or more than 6 hours, SWS\% was more than $25 \%$ and REM\% was more than 20\%. G2: Bad sleepers were those who have less than half of any of the normal values of Sleep EEG, i.e. TST was less than 3 hours, SWS\% was less than $12 \%$ and REM\% was less than 10\%. G3: Moderate sleepers are those who have values between G1 \& G2, i.e. TST between 3-6 hours, SWS\% between 12\%- 25\% and REM\% between 10\%-20\%.

Propofol requirements determination: Once reaching the operating theater, all patients were attached to basic monitoring i.e. 5 lead ECG, non-invasive blood pressure monitoring (NIBP), and pulse oximetry. Bispectral index (BIS) electrode was applied before induction (Infinity Kappa, Dräger, and Lübeck, Germany). Induction of anesthesia was done by propofol intravenous administration. The propofol dose required to achieve BIS value between 40 and 60 was recorded for each patient in $\mathrm{mg}$. Since the weight of each patient was predetermined in $\mathrm{kg}$, the dose was recorded as $\mathrm{mg} / \mathrm{kg}$. 
Statistical analysis: Pearson correlation between propofol dose and both SWS \% and REM \% was done in all patients $(n=27)$. Correlation was considered significant if $\mathrm{p}$ value was $\leq 0.01$. Comparison between the median dose of propofol induction dose in good sleepers $(n=10)$ and bad sleepers $(n=9)$ was done using Mann-Whitney $\mathrm{U}$ test. The data were considered significant if $\mathrm{p}$ value was $\leq 0.05$. Statistical analysis was performed with the aid of the SPSS computer program (version 12 windows).

\section{Results}

There was a positive correlation between propofol induction dose $\mathrm{mg} / \mathrm{kg}$ and both SWS \%, $r=0.532(\mathrm{p}=0.003)$ and REM $(\%)$ $\mathrm{r}=0.518(\mathrm{p}=0.005)$ in patients.

The patients $(n=27)$ were then divided into three groups: Good sleepers $(n=10), B a d$ sleepers $(n=9)$ and moderate sleepers group $(\mathrm{n}=8)$.

There was no significant difference between good and bad sleepers regarding age $\&$ BMI. Mean good sleepers' age was (30.25 \pm 9.41$)$, mean bad sleepers' age was (29.95 \pm 9.34$)(p>0.05)$, mean good sleepers' BMI was (28.65 \pm 3.63$)$, and mean bad sleepers' BMI was $(29.55 \pm 3.50)(\mathrm{p}>0.05)$. The median propofol dose $(\mathrm{mg} / \mathrm{kg})$ for induction of general anesthesia in bad sleepers 1.10 (0.80-1.70) was significantly lower than the median induction dose of propofol in good sleepers $1.70(1.00-2.00 ; \mathrm{P}=0.01)$. Details were given in table (1) and figures (1, 2 \& $3)$.

\section{Discussion}

Although not identical, sleep and anesthesia have similarities as hypnosis, amnesia, immobility and reduced response to external stimuli (Lydic et al, 2005). These similarities are supported by data as similar regional changes in brain activity by labeled positron emission tomography (PET) during-anesthesia and sleep (Alkire et al, 1999). Bispectral index scale can monitor depth of both anesthesia and sleep in patients without sleep disorders (Sleigh et al, 1999) (Tung et al, 2002). Anesthetics administered into brain areas responsible for sleep regulations produced anesthesia (Tung et al, 2001). Moreover Intraoperative dreaming experiences were correlated with dream features in natural sleep (Cascella et al, 2017).

Differences between states of anesthesia and sleep include ability of sleep only to meet physiological needs, which may be due to rapid eye movement sleep (REM) (Mashour et al, 2010). Also unlike anesthesia, sleep is initiated and regulated by many endogenous homeostatic mechanisms and have clearly defined behavioral and physiological endpoints (Carskadon and Dement, 1994).

Regarding propofol and sleep specifically, several recent studies proposed a serious hypothesis that propofol appears to satisfy the need for both REM and SWS (Pick et al, 2011) (Pal et al, 2001). By analyzing those studies, it was noticed that all of them depended on animal models especially rats. Moreover, their argument in confirming their hypothesis was based on (sleep dept) monitoring and induced 24 hours sleep deprivation. Although these approaches were insightful yet, unfortunately, they cannot be implemented on humans. Hence, a natural, simple and non-invasive approach is needed. In this study, a new perspective is offered in investigating propofol sleep interaction. Up to the authors' knowledge, this is the first study investigating the relation between propofol and sleep in humans under no induced abnormal conditions. Also, from this new perspective an important clinical impact was extracted.

First, the induction dose of propofol was strongly positively correlated with SWS\% and REM\% at the night of surgery. In principle, preoperative sleep can affect propofol induction dose in three ways (no correlation, negative correlation and positive correlation). No correlation means that they have two completely independent mechanisms. Being correlated, this supports the fact that the mechanism of action of propofol and natural sleep share common physiological pathway. Nevertheless, if both were posi- 
tively correlated, i.e. the less the REM\% and SWS \% the less the dose of propofol needed for induction of general anesthesia, this will mean that bad sleep quality at night of surgery increased the propensity to general anesthesia induction by propofol. Furthermore, it is known that even mild sleep loss increases the propensity to natural sleep (Bonnet, 1994). This fact supports more the finding that natural sleep and propofol share common pathways in the human brain. If negative correlation was found i.e. bad sleep quality at the night before surgery increases propofol dosing for induction of general anesthesia, this would have meant that not only both share common pathway but also both are affected by the same stimuli i.e. anxiety. It is worth noting that the most common cause of acute sleep deprivation at the night of surgery in a person with no history of sleep disturbances is most probably anxiety. The possible common pathways between sleep and propofol are: Sleep deprivation is proven to increase extracellular adenosine concentrations in the basal forebrain of experimental animals, which delays recovery from general anesthesia (Tanase et al, 2003). Extracellular adenosine concentration is believed to play a role in control mechanisms for both sleep and anesthesia. Reduction in thalamic activity was noticed during anesthesia and naturally occurring sleep (Vahle et al, 2001; Reed and Plourde, 2015).

Second, from this correlation we tried to extract clinical impact and answer a proposed question about whether preoperative sleep quality can affect the propofol induction dose? So, the induction dose of propofol in good and bad sleepers was compared. The propofol requirements to induce general anesthesia in bad sleepers was significantly lower than good sleepers.

This result should alert anesthesiologist to add preoperative sleep quality to their routine history taking. Bad preoperative sleep quality was a common condition due to anxiety, so the present finding helps adjusting propofol dose to meet patients' requirements and avoiding unwanted hypotension, bradycardia and apnea associated with propofol administration. Furthermore, this finding may be a factor affecting propofol dosing in total intravenous anesthesia (TIVA) thus helping in having better postoperative recovery.

To assess sleep quality, usually sleep questioners were used, e.g. Stanford Sleepiness Scale (Hoddes et al, 1972) and epworth sleepiness scale. Unfortunately, these questioners were very subjective and depend on many variables. Alternatively, sleep EEG offered better objective numerical data for many sleep parameters. This study proposed an EEG based classification of good and bad sleep quality. Since restoration of brain energy, information processing and memory formation occurs mainly in SWS and REM

(Guiditta et al, 1995) any suggested classification without including these two parameters would be lagging and there was a strong relation between SWS and sleep continuity and TST (Sugi et al, 2009), so TST was added to classification.

Determination of good sleepers was straight forward as the normal cutoff values of the above mentioned sleep parameters are agreed upon (Sleigh et al, 1999). However, the determination of bad sleepers was somehow challenging because a very wide range of below normal values exists, i.e. normal REM should be equal to or more $20 \%$ of TST so any number from 0 to 19 is considered abnormal. As sleep is an active integrative process with so many compensatory mechanisms not all sleep parameters should be within normal range to ensure good sleep quality. To overcome this problem a third group of patients with intermediate values was created and retrospectively it was eliminated from propofol dose comparison. This creates better identified good and bad sleepers groups and ensures accurate results.

In this study, since the target was to assess propofol relation with sleep homeostasis in humans under natural conditions patients with ESS scores more than 5 were not ini- 
tially included. This ensured that all patients had the same level of sleepiness. Having sleep EEG picture usually completely different than that of normal ones, it was important to eliminate any patient with either symptoms or ESS scores suggesting chronic sleep deprivation. Sleep onset REM, severe knock out in SWS\% as well as frequent arousals and awakening are common EEG findings in such patients (Sugi et al, 2009). It is worth noting that obstructive sleep apnea, insomnia and depression were the most common causes of chronic sleep deprivation and excessive daytime sleepiness. Female gender was also excluded due to a cultural issue, as it is not easily acceptable for a male EEG technician to stay all night beside a sleeping female (no female technicians were available at the study time). Though studying only male might be considered a limitation to this study, it was never hypothesized that the mechanisms of action of propofol and physiological sleep differs between male and female sex.

Table 1: Demographic data and propofol dose in study groups.

\begin{tabular}{|l|c|c|}
\hline Variable & Good sleepers $\mathrm{n}=10$ & Bad Sleepers $\mathrm{n}=9$ \\
\hline Age & $30.25 \pm 9.41$ & $29.95 \pm 9.34$ \\
\hline BMI & $28.65 \pm 3.63$ & $29.55 \pm 3.50$ \\
\hline Propofol dose $(\mathrm{mg} / \mathrm{kg})$ & $1.70(1.00-2.00)$ & $1.10(0.80-1.70)^{*}$ \\
\hline
\end{tabular}

Age \& BMI as mean \pm SD, Propofol dose as median and range* $\mathrm{P}$ value $<0.05$

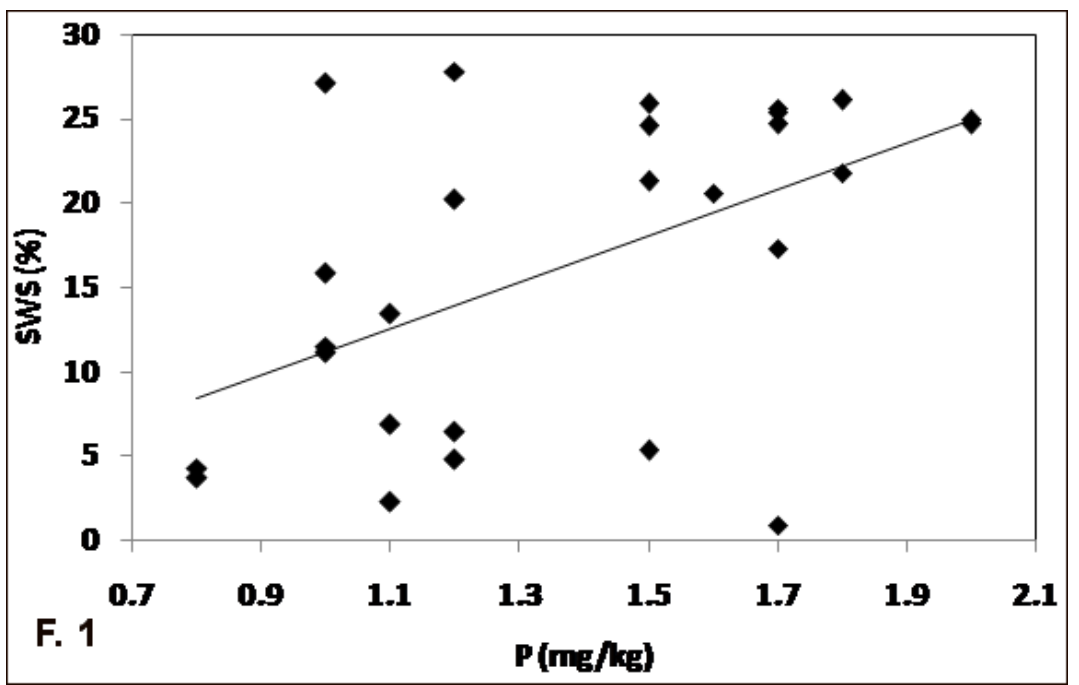

Fig. 1 Correlation between propofol induction dose $(\mathrm{mg} / \mathrm{kg})$ and SWS $(\%)(\mathrm{r}=0.532 ; \mathrm{p}=0.003)$ in patients.

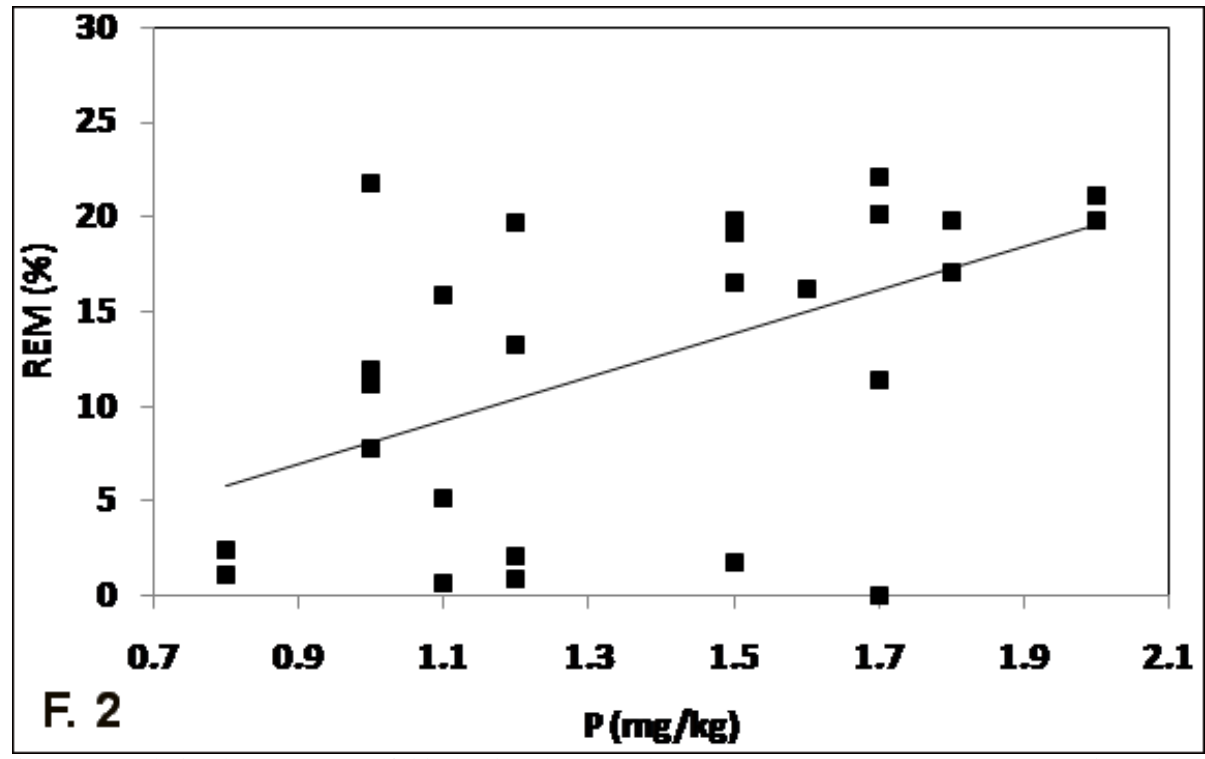

Fig. 2: Correlation between propofol induction dose $(\mathrm{mg} / \mathrm{kg})$ and REM $(\%)(\mathrm{r}=0.518 ; \mathrm{p}=0.005)$ in patients. 


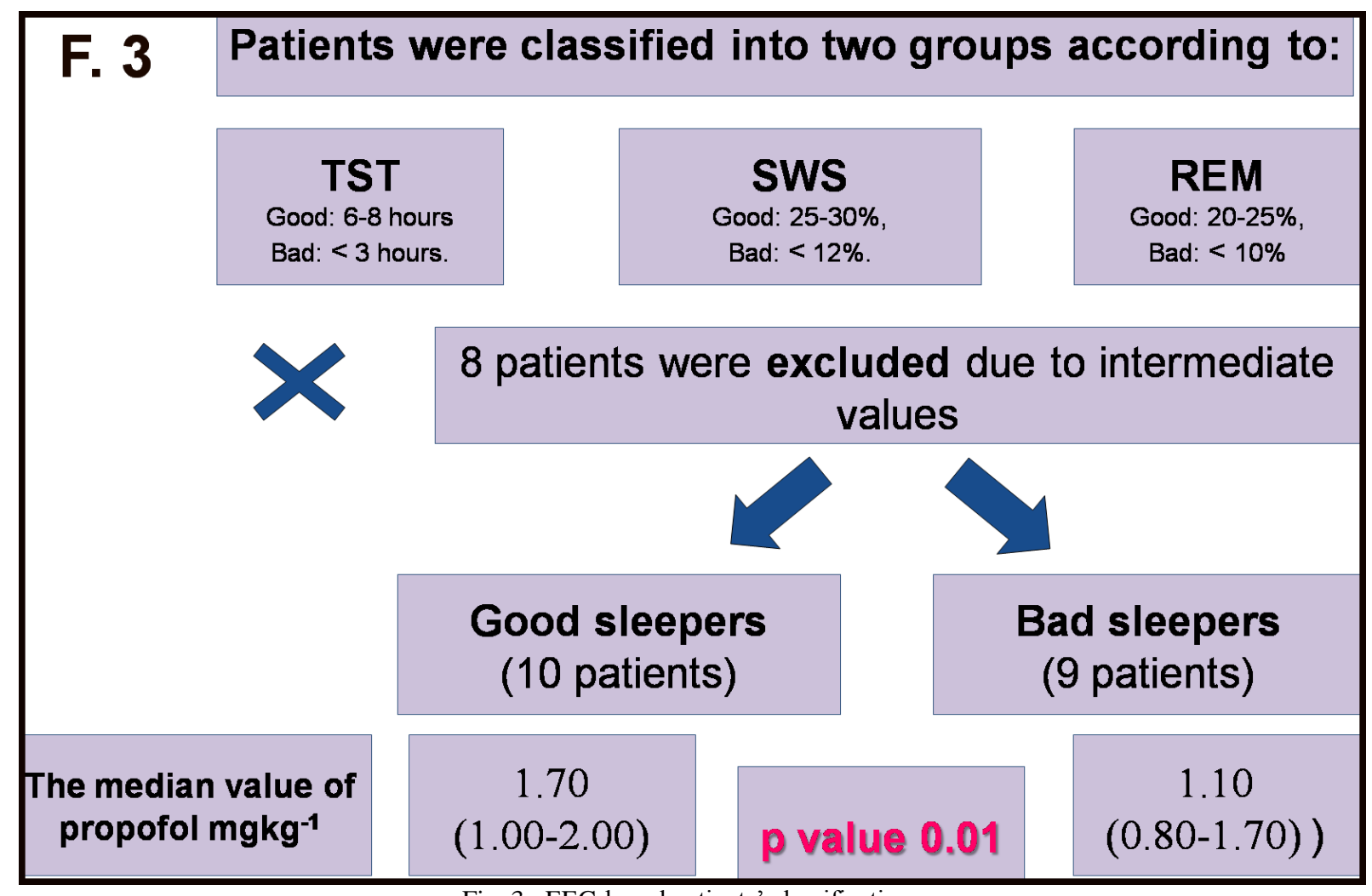

Fig. 3: EEG-based patients' classification.

\section{Conclusion}

Up to the authors' knowledge, this is the first study to confirm the common neurophysiological mechanisms between propofol and REM\% and SWS\% in humans by studying natural sleep under no induced maneuvers. The dose of propofol for induction of general anesthesia in bad sleepers was significantly lower than that of propofol in good sleepers. This finding should alert anesthesiologists to add preoperative sleep quality to their routine history taking and adjusting propofol dose to meet patients' requirements. The study design proved feasible and safe for future full-scale studies to confirm those results.

\section{References}

Alkire, MT, Pomfrett, CJ, Haier, RJ, et al, 1999: Functional brain imaging during anesthesia in humans: Effects of halothane on global and regional cerebral glucose metabolism. Anesthesiology 90:701-9.

Bonnet MH, 1994: Sleep deprivation. In: Principles and Practice of Sleep Medicine. By MH
Kryger, T Roth, WC Dement (eds.): Saunders, Philadelphia.

Brown, EN, Lydic, R, Schiff, ND, 2010: General anesthesia, sleep, and coma. N. Engl. J. Med. 363, 27:2638-50.

Carskadon, MA, Dement, WC, 1994: Normal human sleep. In: Principles and Practice of Sleep Medicine. By MH, Kryger, T, Roth, WC, Dement (eds.): Saunders, Philadelphia.

Cascella, M, Fusco, R, Caliendo, D, Granata, V, Carbone, D, et al, 2017: Anesthetic dreaming, anesthesia awareness and patient satisfaction after deep sedation with propofol target controlled infusion: A prospective cohort study of patients undergoing day case breast surgery. Oncotarget.. doi: 10.18632/oncotarget.17238.

[Epub ahead of print]

Chidambaran, V, Costandi, A, D'Mello, A, 2016: Propofol: A review of its role in pediatric anesthesia and sedation. CNS Drugs: Author manuscript; available in PMC 2016 Jul 1.

Franks, NP, 2008: General anaesthesia; from molecular targets to neuronal pathways of sleep and arousal. Nature Rev. Neurosci. 9:370-86.

Giuditta, A, Ambrosini, MV, Montagnese, P, et al, 1995: The sequential hypothesis of the 
function of sleep. Behavior. Brain Res. 69:15766.

Hoddes, E, Zarcone, V, Dement, W, 1972: Development and use of Stanford Sleepiness scale (SSS). Psychophysiol. 9:150-6.

Kaitlyn, L, Maier, Andrew R. McKinstryWu, Ben Julian A. Palanca, et al, 2017: Protocol for the Reconstructing Consciousness and Cognition (ReCCognition) Study. Front Hum. Neurosci. 11:284-9.

Lydic, R, Baghdoyan, HA, 2005: Sleep, anesthesiology, and the neurobiology of arousal state cont-rol. Anesthesiology 103:1268-85.

Mashour, GA, Lipinski, WJ, Matlen, LB, et al, 2010: Isoflurane anesthesia does not satisfy the homeostatic need for rapid eye movement sleep. Anesth. Analgesia 110:1283-91.

Murphy, M, Bruno, MA, Riedner, BA, et al, 2011: Propofol anesthesia and sleep: a highdensity EEG study. Sleep 34:283-8.

Ohayon, MM, Carskadon, MA, Guilleminault, C, Vitiello, MV, 2004: Meta-analysis of quantitative sleep parameters from childhood to old age in healthy individuals: developing normative sleep values across the human life-span. SleepNew York Then Westchester 27: 1255-74.

Pal, D, Lipinski, WJ, Walker, AJ, Turner, A M, Mashour, GA, 2001: State-specific effects of sevoflurane anesthesia on sleep homeostasis: selective recovery of slow wave but not rapid eye movement sleep. Anesthesiology 114:30210.

Pick, J, Chen, Y, Moore, JT, Sun, Y, Wyner, AJ, et al, 2011: Rapid eye movement sleep debt accrues in mice exposed to volatile anesthetics. Anesthesiology 115:702-9.

Reed, SJ, Plourde, G, 2015: Attenuation of high-frequency $(50-200 \mathrm{~Hz})$ thalamocortical EEG rhythms by propofol in rats is more pronounced for the thalamus than for the cortex. PLoS One 15, 4:10-8.

Sleigh, JW, Andrzejowski, J, Steyn-Ross, A, Steyn-Ross, M, 1999: The bispectral index: A measure of depth of sleep? Anesth. Analgesia 88:659-61.

Sugi, T, Kawana, F, Nakamura, M, 2009: Automatic EEG arousal detection for sleep apnea syndrome. Biomed. Signal Process. Control 4: 329-37.

Tanase, D, Baghdoyan, HA, Lydic, R, 2003: Dialysis delivery of an adenosine A1 receptor agonist to the pontine reticular formation decreases acetylcholine release and increases anesthesia recovery time. Anesthesiology 98:912-20. Tung, A, Bluhm, BB, Mendelson, WB, 2001: The hypnotic effect of propofol in the medial preoptic area of the rat. Life Sci. 69:855-62.

Tung, A, Lynch, JP, Mendelson, WB, 2001: Prolonged sedation with propofol in the rat does not result in sleep deprivation. Anesth. Analgesia 92:1232-16.

Tung, A, Lynch, JP, Roizen, MF, 2002: Use of the BIS monitor to detect onset of naturally occurring sleep. J. Clin. Monit. Comput. 17:37-42.

Tung, A, Mendelson, WB, 2004: Anesthesia and sleep. Sleep Med. Rev. 8:213-25.

Tung, A, Szafran, MJ, Bluhm, B, Mendelson, WB, 2002: Sleep deprivation potentiates the onset and duration of loss of righting reflex induced by propofol and isoflurane. Anesthesiology 97:906-11.

Tung, A, Bergmann, BM, Herrera, S, Cao, D, Mendelson, WB, 2004: Recovery from sleep deprivation occurs during propofol anesthesia. Anesthesiology-Philadelphia Then Hagerstown 100:1419-26.

Vahle-Hinz, C, Detsch, O, Siemers, M, Kochs, E, Bromm, B, 2001: Local GABAA receptor blockade reverses isoflurane's suppressive effects on thalamic neurons in vivo. Anesth. Analgesia 92:1578-84.

Weber, B, Schaper, C, Bushey, D, et al, 2009: Increased volatile anesthetic requirement in short-sleeping Drosophila mutants. Anesthesiology 110:313-8. 